\title{
RESERVORIO DE CARBONO EN SUELO Y RAÍCES DE UN PASTIZAL Y UNA PRADERA BAJO PASTOREO
}

\author{
CARBON STOCK IN SOIL AND ROOTS OF A GRASSLAND AND A SWARD UNDER GRAZING
}

\author{
Flora E. Céspedes Flores ${ }^{1 *}$, Juan A. Fernández ${ }^{1}$, José A. Gobbi ${ }^{2}$ y Aldo C. Bernardis ${ }^{1}$
}

\begin{abstract}
${ }^{1}$ Facultad de Ciencias Agrarias. Universidad Nacional del Nordeste (UNNE). Sargento Cabral 2131. Tel. +54 (03783) 427589 int. 145.3400 , Pcia. Corrientes,

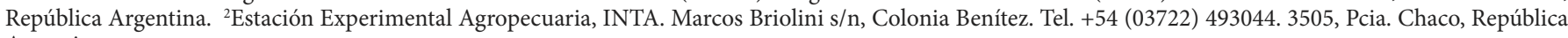
Argentina.

“Autor para correspondencia (fcespedes@agr.unne.edu.ar)
\end{abstract}

\section{RESUMEN}

El objetivo del trabajo fue evaluar el efecto del pastoreo animal sobre el almacén de $\mathrm{C}$ orgánico en suelo y raíces de un pastizal de Sorghastrum setosum y de una pradera de Cynodon nlemfluensis. El estudio se realizó en General Obligado y en Colonia Benítez, en Chaco, Argentina. Se tomaron muestras de suelo y biomasa radical de cuatro profundidades: 0-0.1 m, 0.1-0.2 $\mathrm{m}, 0.2-0.6 \mathrm{~m}$ y 0.6-1 $\mathrm{m}$. El C en suelo se determinó por el método de oxidación de la materia orgánica, y la masa neta de $\mathrm{C}$ se estimó con la densidad aparente en cada estrato. Las raíces fueron lavadas y separadas del suelo con tamices, y se secaron a $65^{\circ} \mathrm{C}$ para determinar materia seca. El C se calculó al asumir un 0.45 de $\mathrm{C}$ en la MS. El diseño estadístico fue un diseño en bloques al azar con cinco bloques en el pastizal y tres en la pradera, con dos tratamientos de pastoreo (con y sin). El contenido de $\mathrm{C}$ total acumulado en suelo a $1 \mathrm{~m}$ fue de $110.1 \mathrm{Mg} \mathrm{ha}^{-1}$ en el pastizal y de $108.6 \mathrm{Mg} \mathrm{ha}^{-1}$ en la pradera, con diferencias $(P<0.05)$ entre los estratos de $0.2 \mathrm{~m}$ y $0.6 \mathrm{~m}$ en ambos sitios. El contenido de $\mathrm{C}$ acumulado en suelo disminuye con la profundidad y más de la mitad del $\mathrm{C}$ se encuentra en los primeros $0.2 \mathrm{~m}$ en ambos sitios. El C acumulado en raíces fue similar en ambos sitios, con diferencias $(P<0.05)$ entre los dos estratos extremos. La menor masa de raíces fue a $1 \mathrm{~m}$, mientras que su mayor volumen y distribución fue hasta los $0.2 \mathrm{~m}$.

Palabras clave: Sorghastrum setosum, Cynodon nlemfluensis, pastoreo, stock de carbono.

\section{SUMMARY}

This study was done was to evaluate the effect of animal grazing on organic C storage in soil and roots in a Sorghastrum setosum grassland and a Cynodon nlemfluensis sward. The study was carried out at Campo Anexo General Obligado and EEA INTA Colonia Benítez, in Chaco, Argentina. Soil and root biomass were sampled at four depths: 0-0.1 $\mathrm{m}, 0.1-0.2 \mathrm{~m}, 0.2-0.6 \mathrm{~m}$ and $0.6-1 \mathrm{~m}$. C in soil was determined by the oxidation of organic matter method, and the $C$ net mass was estimated according to the bulk density at each soil depth. Roots were washed and separated from the soil using sieves, and the samples were oven dried at $65{ }^{\circ} \mathrm{C}$ for dry matter. The $\mathrm{C}$ was calculated assuming a 0.45 fraction. The experimental design was a randomized block design with five blocks in the grassland and three in the sward, with two grazing treatments (with and without). Cumulative soil $\mathrm{C}$ content up to $1 \mathrm{~m}$ depth was 110.1 and $108.61 \mathrm{Mg} \mathrm{ha}^{-1}$ for the grassland and the sward, respectively, with significant differences $(P<0.05)$ between layers at 0.2 and $0.6 \mathrm{~m}$ at both sites. Soil C content decreased with soil depth, and more than half of the $\mathrm{C}$ was found at the first $0.2 \mathrm{~m}$ at both sites. Accumulated $\mathrm{C}$ in roots was similar at both sites, with significant differences $(P<0.05)$ between the two extreme strata. The lowest root mass was at $1 \mathrm{~m}$ depth, while its higher volume and distribution was up to $0.2 \mathrm{~m}$.

Index words: Sorghastrum setosum, Cynodon nlemfluensis, grazing, carbon stock.

\section{INTRODUCCIÓN}

Los suelos son la mayor fuente y reservorio de carbono (C) en los ecosistemas terrestres, y son la vía principal por la cual el $\mathrm{CO}_{2}$ fijado por las plantas es retornado a la atmósfera. La emisión de $\mathrm{CO}_{2}$ de los suelos a la atmósfera ocurre principalmente por la respiración de raíces y organismos heterótrofos (Palacio y Hurtado, 2008).

En la mayoría de zonas agrícolas tropicales se ha observado un severo agotamiento de las reservas de $\mathrm{C}$ en el suelo por efecto de actividades humanas. Se calcula que algunos suelos en las zonas agrícolas tropicales han perdido de 20 a $80 \mathrm{Mg} \mathrm{ha}^{-1}$ de C, en su mayoría liberado a la atmósfera (Lal, 2004). La disminución del nivel de $\mathrm{C}$ orgánico de los suelos se explica por el uso agrícola y la deforestación, ya que la materia orgánica se disminuye principalmente en la capa de suelo labrada debido a una caída de aportes de residuos, al incremento de temperatura y a la destrucción de macro y micro-agregados (Trumper et al., 2009).

Una contribución importante para abatir el aumento de $\mathrm{CO}_{2}$ en la atmósfera es incrementar los reservorios de C edáfico, el cual tiene tiempos medios de residencia más prolongados que la vegetación. En este sentido, las tierras de pastoreo juegan un papel importante en el secuestro de C, ya que los pastizales contribuyen a mitigar el cambio climático global al almacenar $\mathrm{C}$ en la biomasa por el proceso de la fotosíntesis y en el suelo por el ciclo del C (Conant et al., 2005). No obstante, se han efectuado pocas investigaciones sobre especies subtropicales de pastizales 
como potenciales reservas de $\mathrm{C}$ en el suelo, y son menos aún los que evalúan el efecto de tratamientos de manejo sobre el mismo (Lal, 2004).

Según la FAO (2002), las tierras de pastoreo ocupan 3200 millones de hectáreas y almacenan entre 200 y 420 miles de millones de $\mathrm{Mg}$ de $\mathrm{C}$ en el ecosistema total, gran parte debajo de la superficie y en un estado relativamente estable. El C del suelo en tierras de pastoreo es estimado en $70 \mathrm{Mg}$ ha $^{-1}$, cifra similar a las cantidades almacenadas en los suelos forestales (Trumbmore et al., 1995).

Existe poca información del reservorio de $\mathrm{C}$ en los suelos de pastizales, por lo cual el presente estudio se llevó a cabo con el objetivo de evaluar el efecto del pastoreo animal sobre el almacén de $\mathrm{C}$ en el suelo y raíces de un pastizal de Sorghastrum setosum (Griseb.) Hitchc y de una pradera de Cynodon nlemfluensis Vanderyst en el Chaco Argentino.

\section{MATERIALES Y MÉTODOS}

Para poder hacer un análisis comparativo del efecto de ganado bovino en un sistema ganadero, la experiencia se llevó a cabo en dos sitios: un pastizal natural dominado por Sorghastrum setosum, y el otro una pradera de Cynodon nlemfluensis. En ambos ensayos se mantuvo constante el manejo de cargas constante, que son típicas para cada tipo de sistema.

\section{Descripción de las áreas en estudio}

El ensayo del pastizal de Sorghastrum setosum (paja amarilla) fue conducido un campo ubicado en General Obligado, a $59^{\circ} 24^{\prime} \mathrm{LO}$ y $27^{\circ} 19^{\prime}$ de LS, en la provincia del Chaco, República Argentina. El campo se encuentra en el parque Chaqueño oriental (Chaco húmedo). Su clima es húmedo y las precipitaciones aumentan de oeste a este. La temperatura media anual es de 19 a $23{ }^{\circ} \mathrm{C}$, con máximas en verano de $40{ }^{\circ} \mathrm{C}$ y mínimas de $3{ }^{\circ} \mathrm{C}$ en invierno; las condiciones de horas luz (fotoperíodo) son elevadas especialmente hacia el oeste (Cabrera, 1994). De acuerdo con la caracterización realizada por Ledesma y Zurita (1995), el suelo corresponde a la Serie Charadai, es un natracualf típico con gran retención de agua hasta los 170 $\mathrm{cm}, \mathrm{pH}$ ácido en superficie, neutro en profundidad, rico en calcio; su textura es arcillosa, montmorillonítica, de permeabilidad lenta.

El área que comprende el ensayo de pastizal es un campo natural con pendiente de norte a sur (1\%). En las partes altas de lomerío predominan las especies Elionurus muticus (Spreng) Kuntze, Panicum sp.; en la media loma hay Sorghastrum setosum, y en el área lindante con el bajo, con mayor dinámica hídrica, está el pajonal de
Paspalum intermedium Munro ex Morong \& Britton y especies características de los bajos inundables, como el complejo Leersia hexandra Sw. y Luziola peruviana Gmelin, considerando al pajonal como un conjunto de especies vegetales con una especie dominante (Bernardis et al., 2005). En todo el perfil topográfico se observan áreas de transición entre un tipo de vegetación y otro.

El pastizal estudiado no posee historial de pastoreo, de modo que ésta es la primera vez que se evalúa el ingreso de animales en este pastizal. Se utilizaron 0.33 unidades de ganado en una hectárea (UG ha-1) como carga permanente de animales. Se definieron en este sitio cinco potreros de 50 $\mathrm{m}^{2}$, y cada uno representó un bloque con dos tratamientos $(\mathrm{T} 1=\sin$ pastoreo, $\mathrm{yT} 2=$ con pastoreo $)$. En cada tratamiento se tomaron muestras de suelo y raíces en cuatro estratos de profundidad. Los muestreos de suelo se hicieron al inicio en primavera y al final de invierno (fin del ensayo), para así abarcar las cuatro estaciones del año.

Se hizo un ensayo paralelo a la prueba de S. setosum, con una carga animal alta instantánea sobre una pastura de Cynodon nlemfluensis (pasto Estrella), en una parcela ubicada en Colonia Benítez, a $58^{\circ} 56^{\prime} 38^{\prime \prime}$ LO y $27^{\circ} 18^{\prime}$ $26^{\prime \prime}$ LS. Su clima es húmedo, con precipitaciones promedio de $1300 \mathrm{~mm}$ anuales, temperatura media anual de $21.5^{\circ} \mathrm{C}$ y un periodo libre de heladas de 340 a $360 \mathrm{~d}$. El suelo del sitio está representado por el complejo de la Serie Río Negro y Serie Zorrilla (Ledesma y Zurita, 1995). Ambas series tienen alta capacidad de retención de agua, con problemas de acidez y anegabilidad, sin movimiento de agua en el perfil. La serie Río Negro es de textura arcillosa, en tanto que la serie Zorrilla presenta una textura media o franca.

Este sitio posee un historial de pastoreo de 10 años, con tres potreros, y cada uno con una carga animal instantánea de $3.81 \mathrm{UG} \mathrm{ha}^{-1}$, con turnos de $7 \mathrm{~d}$ de pastoreo con $35 \mathrm{~d}$ de descanso. Como tratamiento sin pastoreo se demarcó un sector dentro de cada potrero de la pastura (al cual no tienen acceso los animales). Cada potrero representa un bloque, y el conjunto de bloques se conformó de manera similar al ensayo de pastizal, con dos tratamientos $(\mathrm{T} 1=\sin$ pastoreo, y T2 $=$ con pastoreo). También aquí se evaluaron cuatro estratos de profundidad en cada tratamiento, donde se tomaron muestras de suelo al inicio y al final del ensayo.

\section{Variables medidas}

Las muestras de suelo y raíces fueron tomadas en el pastizal (General Obligado), de los cinco bloques de $50 \mathrm{~m}^{2}$ cada uno correspondientes a ambos tratamientos, T1 y T2. El mismo criterio se utilizó en el ensayo con pastura (Colonia Benítez), donde se tomaron muestras de tres bloques, con una 
superficie promedio de 0.69 ha por bloque. Las muestras de suelo y raíces se colectaron en cuatro estratos en las calicatas o excavaciones de cada bloque, al inicio (primavera del año 2007) y al final del ensayo (invierno del año 2008). En el pastizal natural se obtuvieron cuatro muestras por bloque y 20 muestras por tratamiento, en los cinco bloques con $\mathrm{T} 1$ y T2 (cinco clausuras sin pastoreo, y cinco con pastoreo), para un total de 80 muestras, 40 al inicio y 40 al final del ensayo. De manera similar se procedió en la pradera donde se obtuvieron 12 muestras por tratamiento, para un total de 24 muestras al inicio y 24 al final del ensayo.

Para la determinación del contenido de $\mathrm{C}$ acumulado en el suelo en el pastizal y la pradera, las muestras se colectaron de calicatas de $1 \mathrm{~m}$ de profundidad. En el perfil de suelo se definieron cuatro profundidades de muestreo: $0-0.1 \mathrm{~m}$, 0.1-0.2 m, 0.2-0.6 m, y 0.6-1 m. Para ajustar el contenido de $C$ acumulado según la densidad aparente (DA) en cada estrato de muestreo, se determinó la DA del suelo mediante el uso del cilindro de Kopecky (Prause, 2006). El contenido de $\mathrm{C}$ acumulado en suelo se calculó a partir del contenido de $\mathrm{C}$ orgánico del mismo, y éste se determinó por el método de Walkley-Black (1934). El contenido de C acumulado en suelo se calculó según Da Silva et al. (2004). Los resultados se expresaron en $\mathrm{Mg} \mathrm{ha}^{-1}$ de C.

$$
C=\operatorname{Conc} \times D A \times T \times 10^{-3} \times 10^{4}
$$

Donde:

$C=$ contenido de $\mathrm{C}$ acumulado en suelo $\left(\mathrm{Mg} \mathrm{ha}^{-1}\right)$; Conc = $\mathrm{C}$ acumulado $(\mathrm{kg})$ en $1 \mathrm{Mg}$ de muestra de suelo; $D A=$ densidad de suelo $\left(\mathrm{Mg} \mathrm{m}^{-3}\right) ; T$ = profundidad de la muestra (m); $10^{-3}$ = factor para expresar en $\mathrm{Mg}$ de C; $10^{4}=$ factor para expresar en $\mathrm{Mg} \mathrm{ha}^{-1}$.

Para la determinación del contenido de $\mathrm{C}$ acumulado en raíces, en ambos sitios de muestreo (pastizal y pradera) y en cada estrato de suelo, las raíces colectadas fueron lavadas y separadas del suelo mediante por tamices con malla de 1 $\mathrm{mm}$; luego se llevaron a estufa a $65^{\circ} \mathrm{C}$ hasta alcanzar peso constante para determinar la materia seca (MS). Las raíces gruesas y finas distinguibles a simple vista fueron separadas manualmente; las más finas hasta $1 \mathrm{~mm}$ fueron separadas por tamices (Etchevers et al., 2005), y las raíces $<1 \mathrm{~mm}$ se excluyeron por no diferenciarse de hojas o de MO del suelo (Etchevers et al., 2005; Trumper et al., 2009). El contenido de $\mathrm{C}$ acumulado en la biomasa de raíces se obtuvo al calcular la MS del total de raíces en cada estrato de suelo, y multiplicar la MS por 0.45 (Etchevers et al., 2005).

En ambos ensayos no se tuvieron en cuenta las heces producidas por los animales, motivo por el cual se hace mención sólo a $\mathrm{C}$ acumulado en suelo y en raíces.
El diseño estadístico para ambos ensayos en forma independiente, correspondió a uno de bloques completos al azar con arreglo de parcelas divididas (Steel y Torrie, 1993), con estructuras anidadas dentro del bloque, con dos tratamientos (con y sin pastoreo), cinco repeticiones (bloques) para el pastizal natural y tres en la pradera. Las profundidades de muestreo en suelo están anidadas dentro de cada bloque (potrero), ya que para cada potrero existen cuatro niveles de profundidad, con efecto fijo.

Con los datos obtenidos se hizo un análisis de varianza y comparación de medias a través de la prueba de Tukey a un nivel de $5 \%$ de significancia, con el paquete InfoStat (2009).

\section{RESULTADOS}

\section{C total acumulado en suelo}

En la Figura 1 se representa el stock de $C$ en suelo a cuatro profundidades en los dos sitios de muestreo, el pastizal de S. setosum y la pradera de C.nlemfluensis. El reservorio de $\mathrm{C}$ acumulado en suelo difirió significativamente $(\mathrm{P}<0.05)$ a los $0.2 \mathrm{~m}$ y $0.6 \mathrm{~m}$ en ambos sitios de muestreo, mientras que de 0.6 a $1 \mathrm{~m}$ no hubo diferencia $(\mathrm{P}>0.05)$.

El reservorio o almacén de C total acumulado en suelo hasta $1 \mathrm{~m}$ de profundidad en el pastizal fue de $110.1 \mathrm{Mg}$ $\mathrm{ha}^{-1} \mathrm{y}$ en la pradera fue de $108.61 \mathrm{Mg} \mathrm{ha}^{-1}$, y ambos sitios tuvieron un comportamiento similar en el almacén de C. Respecto al pastizal, el mayor registro $(\mathrm{P} \leq 0.05)$ de $\mathrm{C}$ total acumulado en suelo se obtuvo a $0.1 \mathrm{~m}$, y el menor a $1 \mathrm{~m}$ (Figura 1).

En la pradera el mayor contenido total de $\mathrm{C}$ acumulado en suelo fue a $0.1 \mathrm{~m}$ (Figura 1), y hubo diferencias ( $\mathrm{P}$ $<0.05$ ) entre todos los estratos, excepto entre 0.6 y $1 \mathrm{~m}$. En la pradera, más de $62 \%$ del C acumulado hasta $1 \mathrm{~m}$ se encontró en los primeros $0.2 \mathrm{~m}$ de profundidad. En el pastizal, $41 \%$ del C acumulado se encontró entre 0 y $0.2 \mathrm{~m}$.

En el pastizal de $S$. setosum se observaron diferencias $(\mathrm{P} \leq 0.05)$ entre muestreos para el tratamiento sin pastoreo (T1) a $0.1 \mathrm{~m}$ (Cuadro 1). Sin embargo, no hubo diferencias para el tratamiento con pastoreo (T2) ni para el resto de los estratos muestreados. El mayor registro de $\mathrm{C}$ acumulado fue a $0.1 \mathrm{~m}$ y el menor a $1 \mathrm{~m}$, en ambos muestreos y tratamientos. En cuanto a las tasas neta de acumulación anual de $\mathrm{C}$, las menores se registraron en $\mathrm{T} 2$, en donde en la mayoría de las profundidades hubo una tasa negativa.

Los registros del contenido de $\mathrm{C}$ acumulado en el suelo bajo la pradera indicaron diferencias entre muestreos $(\mathrm{P}<$ 


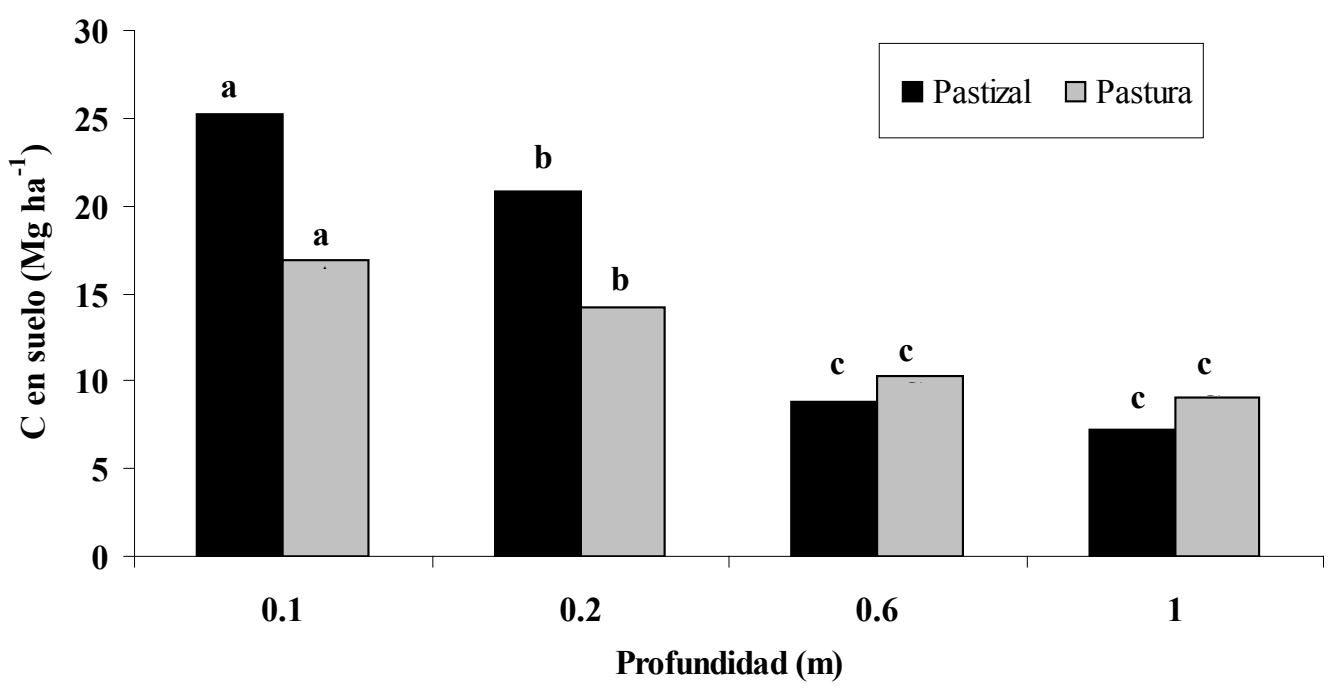

Figura 1. Concentración de $\mathrm{C}$ acumulado en suelo a cuatro profundidades en dos sitios de Chaco, Argentina, pastizal de Sorghastrum setosum (General Obligado) y pradera de Cynodon nlemfluensis (Colonia Benítez). Letras distintas sobre las barras indican diferencias significativas entre profundidades $(\mathrm{P}<0.05)$.

Cuadro 1. Contenido de $\mathrm{C}$ acumulado en suelo $\left(\mathrm{Mg} \mathrm{ha}^{-1}\right)$ a cuatro profundidades, en un pastizal de Sorghastrum setosum (General Obligado), en Chaco, Argentina, al inicio y al final del ensayo, con dos niveles de pastoreo $(\mathrm{T} 1=\sin$; $\mathrm{T} 2=\mathrm{con})$.

\begin{tabular}{|c|c|c|c|c|c|c|c|c|}
\hline \multirow{3}{*}{$\begin{array}{l}\text { Profundidad } \\
\qquad(\mathrm{m})\end{array}$} & \multicolumn{8}{|c|}{ Muestreos General Obligado } \\
\hline & \multicolumn{4}{|c|}{$\mathrm{T} 1$} & \multicolumn{4}{|c|}{$\mathrm{T} 2$} \\
\hline & $\begin{array}{l}\text { Muestreo } \\
\text { Inicial }\end{array}$ & $\begin{array}{l}\text { Muestreo } \\
\text { Final }\end{array}$ & $\begin{array}{c}\text { Tasa de } \\
\text { acumulación } \\
\text { neta anual de C }\end{array}$ & $\begin{array}{l}\text { CV } \\
(\%)\end{array}$ & $\begin{array}{l}\text { Muestreo } \\
\text { Inicial }\end{array}$ & $\begin{array}{c}\text { Muestreo } \\
\text { Final }\end{array}$ & $\begin{array}{c}\text { Tasa de } \\
\text { acumulación } \\
\text { neta anual de C }\end{array}$ & $\begin{array}{l}\text { CV } \\
(\%)\end{array}$ \\
\hline 0.1 & $24.35 \mathrm{a}$ & $29.05 \mathrm{~b}$ & 4.7 & 8.2 & $24.35 \mathrm{a}$ & $24.08 \mathrm{a}$ & -0.27 & 8.4 \\
\hline 0.2 & $19.87 \mathrm{a}$ & $23.58 \mathrm{a}$ & 3.71 & 15.6 & $19.87 \mathrm{a}$ & $21.16 \mathrm{a}$ & 1.29 & 14.1 \\
\hline 0.6 & $9.04 \mathrm{a}$ & $8.52 \mathrm{a}$ & -0.52 & 10.5 & $9.03 \mathrm{a}$ & $8.56 \mathrm{a}$ & -0.48 & 10.5 \\
\hline 1.0 & $6.97 \mathrm{a}$ & $7.47 \mathrm{a}$ & 0.5 & 4.5 & $6.97 \mathrm{a}$ & $7.30 \mathrm{a}$ & 0.33 & 1.4 \\
\hline
\end{tabular}

Letras distintas en una fila indican diferencias significativas entre muestreos (Tukey, 0.05). CV = coeficiente de variación.

0.05) a todas las profundidades, con mayor acumulación de $\mathrm{C}$ mayor en el muestreo final que en el inicial, en todos los estratos (Cuadro 2). Para el T2 se observaron diferencias entre muestreos $(\mathrm{P}<0.05)$ a $0.1,0.2$ y $1 \mathrm{~m}$ de profundidad, con menores valores de $\mathrm{C}$ en el muestreo final. De manera similar a lo registrado en el pastizal, en la pradera de $C$. nlemfluensis las menores tasas netas de acumulación de C se presentaron en el tratamiento con pastoreo y en todas las profundidades hubo una tasa negativa.

\section{$\mathrm{C}$ acumulado en biomasa de raíces}

El contenido de $\mathrm{C}$ acumulado en raíces se comportó de forma similar en ambos sitios (Figura 2), con diferencias ( $\mathrm{P}$ $<0.05$ ) entre $0.1 \mathrm{~m}$ y $1 \mathrm{~m}$, y entre 0.1 y $0.6 \mathrm{~m}$ de profundidad.
En los dos sitios el mínimo de raíces se registró a $1 \mathrm{~m}$; en la pradera se observaron sólo al final del ensayo con valores promedios de $0.05,0.08$ y $0.12 \mathrm{Mg} \mathrm{ha}^{-1}$ de C para los tres bloques. En el pastizal las raíces a $1 \mathrm{~m}$ de profundidad sólo se observaron en los bloques 1 y 2 al inicio del ensayo, con valores de $0.05 \mathrm{Mg} \mathrm{ha}^{-1}$ de $\mathrm{C}$, y al final del ensayo sólo en los bloques 2 y 5 con valores de 0.08 y $0.07 \mathrm{Mg} \mathrm{ha}^{-1} \mathrm{de} \mathrm{C}$, en promedio de tratamientos de pastoreo.

En cuanto al contenido de $\mathrm{C}$ acumulado en raíces en los cuatro estratos muestreados $(0-0.1,0.1-0.2,0.2-0.6$ y $0.6-1$ $\mathrm{m})$, en el pastizal se encontró que en ambos tratamientos de pastoreo (T1 y T2) los coeficientes de variación fueron altos (Cuadro 3), motivo por el cual se dificultó detectar diferencias significativas entre las profundidades de 
muestreo. La mayor cantidad de biomasa de raíces, y por ende mayor registro de $\mathrm{C}$ acumulado en raíces, fue a $0.1 \mathrm{~m}$ y el mínimo a $1 \mathrm{~m}$ de profundidad.

En cuanto al $\mathrm{C}$ acumulado en raíces en la pradera, solamente a $0.1 \mathrm{~m}$ en el tratamiento sin pastoreo (T1) el valor registrado al final superó al inicial (Cuadro 4), mientras que en los estratos inferiores ocurrió lo contrario. En el tratamiento con pastoreo (T2) el C acumulado en raíces siempre fue mayor al inicio que al final del ensayo.

\section{DISCUSIÓN}

\section{C total acumulado en suelo}

La tendencia en ambos sitios fue que más de $60 \%$ del $\mathrm{C}$ acumulado se encontró en los primeros $0.2 \mathrm{~m}$ de suelo, profundidad a la que se encontraba la materia orgánica $(\mathrm{MO})$ en descomposición, resultado esperado porque es la zona donde hay una estratificación de la MO; es decir, el carbono orgánico del suelo activo en constante descomposición y más susceptible a factores externos (FAO, 2002). Zhou et al. (2007) encontraron que en usos del suelo como pastizales y cultivo, los primeros $0.2 \mathrm{~m}$ de profundidad contienen $80 \%$ del C y N del suelo y que $60 \%$ de la MO se almacena sobre los primeros $0.3 \mathrm{~m}$ de suelo; estos autores no observaron diferencias estadísticas en cuanto al almacén de C entre 0.6 y $1 \mathrm{~m}$ de profundidad. Los valores de almacén de $\mathrm{C}$ en el perfil de suelo a $1 \mathrm{~m}$ son similares a los hallados en algunos trabajos de Fisher et al. (1994), ya que a esta profundidad el C almacenado en suelo es menos susceptible a cambios y su modificación es a largo plazo, como lo fundamentaron IPCC (2001), FAO (2002) y Piñeiro et al. (2006).

Se debe considerar que en el pastizal (General Obligado) es el primer año de evaluación del componente animal

Cuadro 2. Contenido de $\mathrm{C}$ acumulado en suelo $\left(\mathrm{Mg} \mathrm{ha}^{-1}\right)$ a cuatro profundidades, en una pradera de Cynodon nlemfluensis (Colonia Benítez), en Chaco, Argentina, al inicio y al final del ensayo, con dos niveles de pastoreo $(\mathrm{T} 1=\sin$; $\mathrm{T} 2=\mathrm{con}$ ).

\begin{tabular}{|c|c|c|c|c|c|c|c|c|}
\hline \multirow{3}{*}{$\begin{array}{l}\text { Profundidad } \\
\text { (m) }\end{array}$} & \multicolumn{8}{|c|}{ Muestreos Colonia Benítez } \\
\hline & \multicolumn{4}{|c|}{$\mathrm{T} 1$} & \multicolumn{4}{|c|}{$\mathrm{T} 2$} \\
\hline & $\begin{array}{l}\text { Muestreo } \\
\text { Inicial }\end{array}$ & $\begin{array}{c}\text { Muestreo } \\
\text { Final }\end{array}$ & $\begin{array}{c}\text { Tasa de } \\
\text { acumulación neta } \\
\text { anual de C }\end{array}$ & $\begin{array}{l}\mathrm{CV} \\
(\%)\end{array}$ & $\begin{array}{l}\text { Muestreo } \\
\text { Inicial }\end{array}$ & $\begin{array}{c}\text { Muestreo } \\
\text { Final }\end{array}$ & $\begin{array}{c}\text { Tasa de } \\
\text { acumulación neta } \\
\text { anual de C }\end{array}$ & $\begin{array}{l}\mathrm{CV} \\
(\%)\end{array}$ \\
\hline 0.1 & $15.68 \mathrm{a}$ & $17.15 \mathrm{~b}$ & 1.47 & 1.7 & $19.21 \mathrm{a}$ & $15.46 \mathrm{~b}$ & -3.75 & 8.6 \\
\hline 0.2 & $14.27 \mathrm{a}$ & $18.95 \mathrm{~b}$ & 4.68 & 1.9 & $13.84 \mathrm{a}$ & $9.63 \mathrm{~b}$ & -4.21 & 6.4 \\
\hline 0.6 & $8.00 \mathrm{a}$ & $10.06 \mathrm{~b}$ & 2.06 & 2.8 & $12.03 \mathrm{a}$ & $11.11 \mathrm{a}$ & -0.92 & 16.2 \\
\hline 1.0 & $10.11 \mathrm{a}$ & $8.47 \mathrm{~b}$ & -1.24 & 2.2 & $10.21 \mathrm{a}$ & $8.30 \mathrm{~b}$ & -1.91 & 2.1 \\
\hline
\end{tabular}

Letras distintas en una fila indican diferencias significativas entre muestreos (Tukey, 0.05 ). CV = coeficiente de variación.

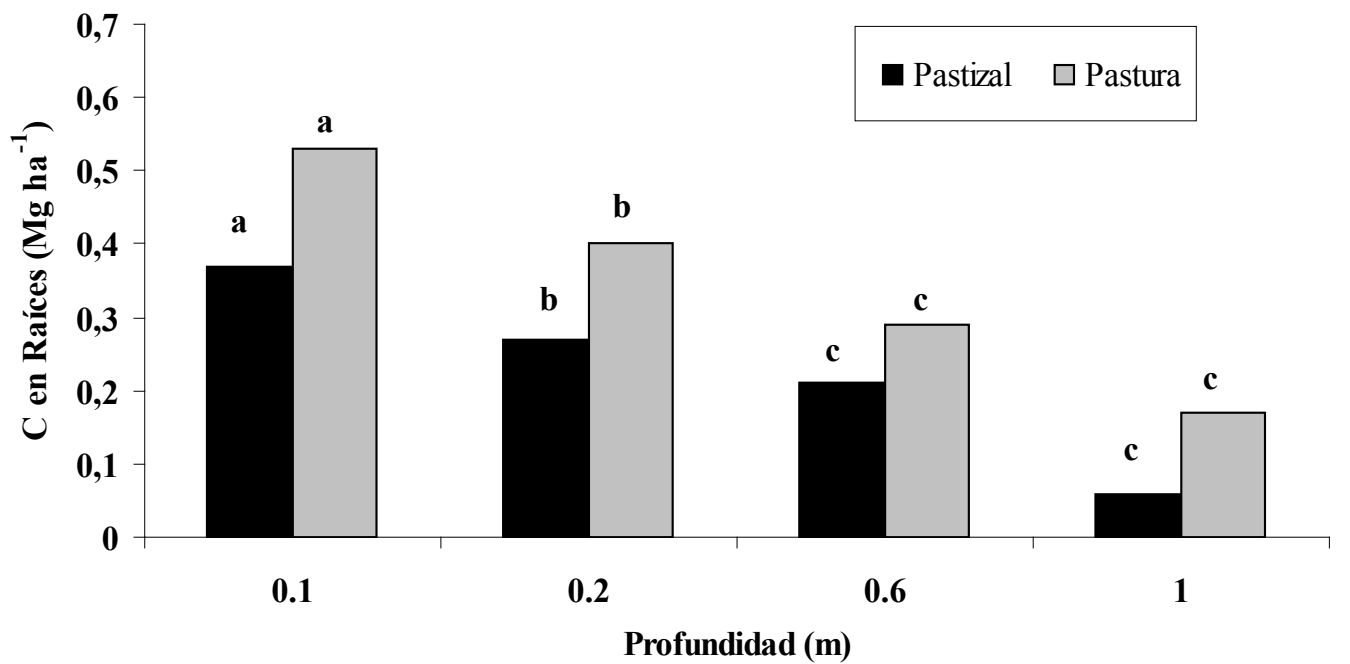

Figura 2. Acumulación de $\mathrm{C}$ en raíces a cuatro profundidades en dos sitios de Chaco, Argentina, en pastizal de Sorghastrum setosum (General Obligado) y en pradera de Cynodon nlemfluensis (Colonia Benítez). Letras distintas sobre las barras indican diferencias significativas entre profundidades $(\mathbf{P}<0.05)$. 
Cuadro 3. Contenido de $\mathrm{C}$ acumulado en raíces $\left(\mathrm{Mg} \mathrm{ha}^{-1}\right)$ a cuatro profundidades del suelo, en un pastizal de Sorghastrum setosum (General Obligado), en Chaco, Argentina, al inicio y al final del ensayo, con dos niveles de pastoreo (T1 = sin; $\mathrm{T} 2$ = con).

\begin{tabular}{|c|c|c|c|c|c|c|}
\hline \multirow{3}{*}{$\begin{array}{l}\text { Profundidad } \\
(\mathrm{m})\end{array}$} & \multicolumn{6}{|c|}{ Muestreos General Obligado } \\
\hline & \multicolumn{3}{|c|}{$\mathrm{T} 1$} & \multicolumn{3}{|c|}{$\mathrm{T} 2$} \\
\hline & $\begin{array}{c}\text { Muestreo } \\
\text { Inicial }\end{array}$ & $\begin{array}{l}\text { Muestreo } \\
\text { Final }\end{array}$ & $\mathrm{CV}(\%)$ & $\begin{array}{l}\text { Muestreo } \\
\text { Inicial }\end{array}$ & $\begin{array}{l}\text { Muestreo } \\
\text { Final }\end{array}$ & $\mathrm{CV}(\%)$ \\
\hline 0.1 & $0.41 \mathrm{a}$ & $0.36 \mathrm{a}$ & 58.5 & $0.41 \mathrm{a}$ & $0.25 \mathrm{a}$ & 69.6 \\
\hline 0.2 & $0.31 \mathrm{a}$ & $0.24 \mathrm{a}$ & 44.5 & $0.31 \mathrm{a}$ & $0.17 \mathrm{a}$ & 45.8 \\
\hline 0.6 & $0.24 \mathrm{a}$ & $0.13 \mathrm{a}$ & 43.7 & $0.24 \mathrm{a}$ & $0.17 \mathrm{a}$ & 39.3 \\
\hline 1 & $0.05 \mathrm{a}$ & $0.11 \mathrm{a}$ & 42.1 & $0.04 \mathrm{a}$ & $0.03 \mathrm{a}$ & 77.2 \\
\hline
\end{tabular}

Letras distintas en una fila indican diferencias significativas entre muestreos (Tukey, 0.05). CV = coeficiente de variación.

Cuadro 4. Contenido de $\mathrm{C}$ acumulado en raíces $\left(\mathrm{Mg} \mathrm{ha}^{-1}\right)$ a cuatro profundidades del suelo, en una pradera de $\mathrm{Cynodon}$ nlemfluensis (Colonia Benítez), en Chaco, Argentina, al inicio y al final del ensayo, con dos niveles de pastoreo (T1 = $\sin ; \mathrm{T} 2$ = con).

\begin{tabular}{|c|c|c|c|c|c|c|}
\hline \multirow{3}{*}{$\begin{array}{l}\text { Profundidad } \\
\qquad(\mathrm{m})\end{array}$} & \multicolumn{6}{|c|}{ Muestreos Colonia Benítez } \\
\hline & \multicolumn{3}{|c|}{ inicial } & \multicolumn{3}{|c|}{$\mathrm{T} 2$} \\
\hline & Muestreo & $\begin{array}{l}\text { Muestreo } \\
\text { final }\end{array}$ & $\mathrm{CV}(\%)$ & $\begin{array}{l}\text { Muestreo } \\
\text { inicial }\end{array}$ & $\begin{array}{l}\text { Muestreo } \\
\text { final }\end{array}$ & $\mathrm{CV}(\%)$ \\
\hline 0.1 & inicial & $0.58 \mathrm{~b}$ & 0.1 & $0.60 \mathrm{a}$ & $0.40 \mathrm{~b}$ & 14.2 \\
\hline 0.2 & $0.88 \mathrm{a}$ & $0.22 \mathrm{~b}$ & 0.5 & $0.34 \mathrm{a}$ & $0.18 \mathrm{a}$ & 57.5 \\
\hline 0.6 & $0.32 \mathrm{a}$ & $0.12 \mathrm{~b}$ & 1.7 & $0.45 \mathrm{a}$ & $0.29 \mathrm{a}$ & 29.4 \\
\hline 1.0 & $0.30 \mathrm{a}$ & $0.05 \mathrm{a}$ & 4.6 & $0.45 \mathrm{a}$ & $0.11 \mathrm{~b}$ & 19.9 \\
\hline
\end{tabular}

Letras distintas en una fila indican diferencias significativas entre muestreos (Tukey, 0.05). CV = coeficiente de variación.

en un sistema de pastoreo, y de acuerdo con los plazos necesarios para observar efecto del pastoreo sobre el $\mathrm{C}$ acumulado en suelo, según la bibliografía consultada, no es esperable encontrar diferencias por el pastoreo animal (IPCC, 2001; Piñeiro et al., 2006). Sin embargo, en el año evaluado si bien los animales no produjeron disturbios en la acumulación del $\mathrm{C}$ en el suelo ya que el pastoreo no causó disminución significativa del $\mathrm{C}$, mientras que en el tratamiento sin pastoreo hubo un aumento del $\mathrm{C}$ acumulado en suelo; pero el pastoreo sí produjo una disminución en la tasa neta anual de acumulación de C. Según los datos del Cuadro 1, en el tratamiento con animales (T2) ocurrieron las menores tasas netas de acumulación de $\mathrm{C}$ en la mayoría de las profundidades de muestreo, lo que indica que el pastoreo afecta la acumulación de C. Según Fang et al. (2010), el pastoreo del ganado y el corte afectan la dinámica del ecosistema de $\mathrm{C}$ en los pastizales de China, debido a que los cambios en el uso del suelo y en el manejo de éste son factores que alteran el equilibrio de la dinámica del C en los pastizales (Zhou et al., 2007).

En la evaluación de los tratamientos de pastoreo (T1 y T2) se debería haber considerado al aporte de material orgánico del mantillo y su tasa de descomposición en superficie y en los primeros estratos de suelo, en ambos sitios estudiados, ya que provee el recurso primario para la formación de MO en el suelo (FAO, 2002; Piñeiro et al., 2006). Las interrogantes que surgirían en este caso es si la carga animal utilizada estimularía una baja producción de mantillo o si las especies bajo análisis harían un aporte significativo de mantillo para lograr un incremento del C orgánico del suelo (Mannetje et al., 2008).

Sin embargo, en la pastura (Colonia Benítez) con un historial de pastoreo de aproximadamente 10 años y una carga instantánea alta de animales, los efectos del pastoreo sobre el contenido de $\mathrm{C}$ acumulado en suelo se observaron al inicio, ya que el tratamiento sin pastoreo presentó un valor menor de $\mathrm{C}$ en suelo respecto al tratamiento con pastoreo, aunque al final del ensayo el tratamiento sin pastoreo tuvo un valor mayor de $\mathrm{C}$ que el tratamiento con pastoreo. La conversión de pastizales a pasturas duplicaría la producción de biomasa aérea e incrementaría el C y el nitrógeno del suelo en 20 \% (Zhou et al., 2007). Valores similares a los nuestros fueron hallados por Goidts y van Wesemael (2007), quienes en pastizales registraron un 
incremento de $21.9 \mathrm{Mg} \mathrm{ha}^{-1}$ del $\mathrm{C}$ en los primeros $0.3 \mathrm{~m} \mathrm{del}$ suelo, sobre un valor inicial de $61.2 \mathrm{Mg} \mathrm{ha}^{-1}$.

La tendencia observada al final del estudio es que el almacén de $\mathrm{C}$ disminuye con el pastoreo ya que los valores del muestreo final son menores a los del muestreo inicial. En cambio, en el tratamiento sin pastoreo el suelo tiende a acumular $\mathrm{C}$ puesto que los valores de $\mathrm{C}$ en el muestreo final son mayores que en el inicial. Esta tendencia no se podría generalizar con datos generados durante un año de estudio, pero sirven para iniciar una base de información que se podría confirmar con estudios a más largo plazo. Según Piñeiro et al. (2006), los cambios ocasionados por el pastoreo son evidentes en evaluaciones de periodos mayores a 50 años. Como mencionan Goidts y van Wesemael (2007), la evolución del almacén de C en el suelo es difícil de evaluar a escala regional debido a la falta de información sobre las prácticas de manejo de los suelos en el presente y en el pasado.

\section{Acumulación de $\mathrm{C}$ en biomasa de raíces}

La utilización de forraje por grandes herbívoros acentúa el interés de conocer el efecto del pastoreo sobre la dinámica de las raíces. La literatura disponible es muy variable y a veces contradictoria, ya que unos casos señala un efecto detrimental del pastoreo sobre las raíces, en magnitud que puede variar en función de la intensidad del pastoreo; y en otros se reporta un efecto benéfico del pastoreo sobre el sistema radical del pastizal, porque estimula el crecimiento del sistema radical al movilizar reservas de fotoasimilados para este fin (Doll y Deregibus, 1986).

Una considerable proporción de material orgánico, por ende de C orgánico, es aportado al suelo por material muerto de raíces y por rizodeposición. De la biomasa radical subterránea aquí observada hasta $1 \mathrm{~m}$ de profundidad en los perfiles de suelo, $67 \%$ y $72 \%$ se encuentra contenida en los primeros $0.2 \mathrm{~m}$ de profundidad tanto en la pradera como en el pastizal natural, lo que indica su distribución superficial en coincidencia con lo observado por Doll y Deregibus (1986), Ansín et al. (1999), Pucheta et al. (2004) y Etchevers et al. (2005).

Como promedio general, aproximadamente $50 \%$ del total de la biomasa de raíces en la pradera se encuentra entre $0.2 \mathrm{~m}$ y $0.4 \mathrm{~m}$ de profundidad, y $75 \%$ en pastizal, lo cual muestra la profundidad del aporte de material de orgánico que realizan estas especies y la profundidad a la cual llegan sus raíces, característica que varía entre especies (Kögel-Knabner, 2002). Zhou et al. (2007) encontraron que $80 \%$ del C contenido en raíces estaba en los primeros $0.2 \mathrm{~m}$ de suelo y que muy pocas raíces estaban por debajo de los $0.5 \mathrm{~m}$ de profundidad, en suelos con pastizales y cultivos agrícolas.

El contenido de humedad en el suelo y la textura en el perfil, entre otros, podrían ser algunos de los factores que indujeron alta variabilidad en la presencia de raíces en el presente estudio, como también señalaron Mannetje et al. (2008), y por ende el de C almacenado en raíces en ambos sitios de muestreo. Por ello en los potreros donde se observaba más humedad en los estratos profundos éstos presentaban mayor presencia de raíces, tanto en el pastizal como en la pradera.

La supresión del pastoreo en el pastizal de S. setosum en la pradera de $C$. nlemfluensis determinó variaciones en la biomasa subterránea durante el ciclo evaluado, ya que fue mayor en el tratamiento sin pastoreo. La pradera, por ser una especie rastrera que posee una gran adaptación al pastoreo, fue más resistente al efecto de una alta carga animal. El pastizal tuvo un comportamiento similar, a pesar de ser una especie de crecimiento erecto y de ser su primer ciclo de pastoreo evaluado (Doll y Deregibus, 1986; Pucheta et al., 2004).

Según Fisher et al. (1994), las especies con sistema radicular profundo constituyen una opción para incrementar en buena medida la acumulación de $\mathrm{C}$, ya que pueden redistribuir el $\mathrm{C}$ en las capas más profundas del suelo. La pradera se mostró homogénea en cuanto a su productividad y a la distribución de las raíces en el perfil a $1 \mathrm{~m}$ de profundidad. El pastizal presentó variabilidad en la distribución de raíces en el perfil de suelo, y por ende el aporte de $\mathrm{C}$ fue variable.

\section{CONCLUSIONES}

En el presente ensayo se encontró que el C acumulado en suelo disminuye a medida que aumenta la profundidad, más de la mitad de este $\mathrm{C}$ se encuentra en los primeros $0.2 \mathrm{~m}$ de profundidad. En el tratamiento con pastoreo el componente animal limitó la acumulación de $\mathrm{C}$ en el horizonte superficial en el pastizal y en la pradera, por lo que disminuyó el reservorio de C. En cuanto al sistema radical, el mayor volumen de raíces se observó a $0.2 \mathrm{~m}$ de profundidad en ambos sitios de muestreo, lo que incrementó el contenido de $\mathrm{C}$ acumulado en el suelo a dicha profundidad, por el aporte de material orgánico que hicieron las raíces. En el pastizal, la variabilidad en los resultados no permitió detectar el efecto del animal sobre la acumulación de $\mathrm{C}$ en la biomasa radical, mientras que en la pradera se observó que el componente animal disminuyó la acumulación de $\mathrm{C}$ en las raíces del estrato más superficial de suelo. 


\section{AGRADECIMIENTOS}

Este trabajo se enmarcó en el Proyecto "Medición de la captura de carbono en sistemas pastoriles representativos del nordeste Argentino" de la Secretaría General de Ciencia y Técnica - Universidad Nacional del Nordeste, y en el Proyecto CHFOR04 INTA Regional Chaco-Formosa (Rep. Argentina).

\section{BIBLIOGRAFÍA}

Ansín O E, E M Oyhamburu, E A Hoffmann, M C Vecchio, M C Ferragine (1999) Distribución de raíces en pastizales naturales y pasturas cultivadas de La Pampa Deprimida Bonaerense y su relación con la biomasa forrajera. Rev. Fac. Agron. La Plata 103:141-148.

Bernardis A C, C A Roig, M Bennasar Vilches (2005) Productividad y calidad de los pajonales de Sorghastrum setosum (grises.) Hitchc. en Formosa, Argentina. Agric. Téc. (Chile) 65:177-185

Cabrera A (1994) Regiones Fitogeográficas Argentinas. Enciclopedia Argentina de Agricultura y Jardinería. Fascículo I. Tomo II. 85 p.

Conant R T, K Paustian, S J Del Grosso, W J Parton (2005) Nitrogen pools and fluxes in grassland soils sequestering carbon. Nutr. Cycling Agroecosyst. 71:239-248.

Da Silva J E, D V S Resck, E J Corazza, L Vivaldi (2004) Carbon storage in clayey Oxisol cultivated pastures in the "Cerrado" region, Brazil. Agric. Ecosyst. Environ. 103:357-363.

Doll U M, V A Deregibus (1986) Efecto de la exclusión del pastoreo sobre el subsistema subterráneo de un pastizal templado húmedo. Turrialba 36:337-344.

Etchevers B J D, C M Monreal, C Hidalgo, M Acosta, J Padilla, R M López (2005) Manual para la Determinación de Carbono en la Parte Aérea y Subterránea de Sistemas de Producción en Laderas. Colegio de Postgraduados, México. 1ra ed. 29 p.

FAO, Food Agricultural Organization (2002) Captura de Carbono en los Suelos para un Mejor Manejo de la Tierra. Informes Sobre recursos Mundiales de Suelos. Organización de las Naciones Unidas para la Agricultura y la Alimentación, Roma. Vol. 96. 70 p.

Fisher M J, I M Rao, M A Ayarza, C E Lascano, I J Sanz, R J Thomas, R R Vera (1994) Carbon storage by introduced deep-rooted grasses in the South American savannas. Nature 371: 236-237.

Goidts E, B van Wesemael (2007) Regional assessment of soil organic carbon changes under agriculture in Southern Belgium (19552005). Geoderma 141:341-354.

InfoStat (2009) Infostat version 1.1. Grupo Infostat, Facultad de Ciencias
Agrarias, Universidad Nacional de Córdoba, Argentina.

Intergovernmental Panel on Climate Change (2001) Glossary of Terms. Synthesis Annex B. Third Assessment Report: Climate Change 2001. Cambridge University Press. Cambridge, U.K. pp:365-388.

Kögel-Knabner I (2002) The macromolecular organic composition of plant and microbial residues as inputs to soil organic matter. Soil Biol. Biochem. 34:139-162.

Lal R (2004) Soil carbon sequestration impacts on global climate change and food security. Science 304:1623-1627.

Ledesma L, J Zurita (1995) Carta de Suelo de la Estación Experimental Agropecuaria Colonia Benítez. E.E.A. R. Sáenz Peña. 164 p.

Mannetje L't, M C Amézquita, P Buurman (2008) Carbon Sequestration in Tropical? Grassland Ecosystems. Wageningen Academic Publishers. 224 p.

Palacio A A R, F H M Hurtado (2008) Respiración microbial y de raíces en suelos de bosques tropicales primarios y secundarios (Porce, Colombia). Rev. Fac. Nal. Agron. Medellín 61:4381- 4393.

Piñeiro G, J M Paruelo, M Oesterheld (2006) Potential long-term impacts of livestock introduction on carbon and nitrogen cycling in grasslands of Southern South America. Global Change Biol. 12:1267-1284.

Prause J (2006) Análisis de Suelos. Técnicas de Muestreos de Suelos, Aguas y Plantas. Bases Prácticas para la Fertilización. $1^{\text {a }}$ ed. Resistencia: Librería de la Paz. 96 p.

Pucheta E, I Bonamici, M Cabido, S Díaz (2004) Below-ground biomass and productivity of a grazed site and a neighboring ungrazed exclosure in a grassland in central Argentina. Austral Ecol. 29:201-208.

Steel R G D, J H Torrie (1993) Bioestadística: Principios y Procedimientos. 2 da ed. México, D.F. McGraw-Hill, Inc., USA . 622 p.

Trumbmore S E, E A Davidson, P Barbosa de Camargo, D D Nepstad, L A Martinelli (1995) Belowground cycling of carbon in forests and pastures of eastern Amazonia. Global Biogeochem. Cycles 9:515-528.

Trumper K, M Bertzky, B Dickson, G Van der Heijden, M Jenkins, P Manning (2009) ¿La Solución Natural? El Papel de los Ecosistemas en la Mitigación del Cambio Climático. Informe del Programa de las Naciones Unidas para el Medio Ambiente. 76 p.

Walkey A, A Black (1934) An examination of the Degjareff method for determining soil organic matter and a proposed modification of the chromic acid titration method. Soil Sci. 37:29-38.

Fang J Y, Y H Yang, W H Ma, A Mohammat, H Shen (2010) Ecosystem carbon stocks and their changes in China's grasslands. Science China-Life Sci. 53:757-765.

Zhou Z, O Sun, J Huang, L Li, P Liu, X Han (2007) Soil carbon and nitrogen stores and storage potential as affected by land-use in an agro-pastoral ecotone of northern China. Biogeochemistry $82: 127-138$. 